\title{
Merkmale des Naturalismus in der österreichischen Antiheimatliteratur
}

Éléments naturalistes dans l'Antiheimatliteratur en Autriche

Naturalist Aspects in the Austrian anti-Aga saga

\section{Sabine Voda Eschgfäller}

\section{(2) OpenEdition}

Journals

Édition électronique

URL : http://journals.openedition.org/austriaca/637

DOI : 10.4000/austriaca.637

ISSN : 2729-0603

Éditeur

Presses universitaires de Rouen et du Havre

Édition imprimée

Date de publication : 1 juin 2018

Pagination : 207-223

ISBN : 979-10-240-1233-9

ISSN : 0396-4590

\section{Référence électronique}

Sabine Voda Eschgfäller, „Merkmale des Naturalismus in der österreichischen Antiheimatliteratur", Austriaca [Online], 86 | 2018, Online erschienen am: 01 Juli 2020, abgerufen am 29 Januar 2021. URL: http://journals.openedition.org/austriaca/637 ; DOI: https://doi.org/10.4000/austriaca.637 


\title{
Merkmale des Naturalismus in der österreichischen Antiheimatliteratur
}

\author{
Heimat gleich (Anti-)Heimat
}

Heimat feiert allerorts, man könnte fast sagen weltweit, ein Comeback (vielleicht war sie auch niemals wirklich passé): Die Politik operiert mit dem Heimatbegriff, Heimat liefert den Hintergrund für immer mehr TV-Serien und figuriert seit Beginn der 200oer-Jahre - Stichwort „Alpenkrimi“ - ebenso erfolgreich in der deutschsprachigen Literatur. ${ }^{1}$

Das (neue?) Genre des „Alpenkrimis“ bzw. der Kriminalliteratur mit regionalem Hintergrund greift auf Traditionen zurück, die - neben mehr oder weniger prominenten Merkmalen der Detektivliteratur - auch aus dem Repertoire der Heimat- und Antiheimatliteratur stammen. Die Tendenz dabei geht anscheinend in die Richtung, die Anteile der Antiheimatliteratur mehr und mehr zu reduzieren, um eine einfachere „Konsumierbarkeit“ durch das breite Publikum zu erreichen.

Es handelt sich hier also um eine Entwicklung, die auf den ersten Blick polar entgegengesetzte Phänomene wie die Heimat- und die Antiheimatliteratur $^{2}$ für die eigenen Zwecke domestiziert und vor einen

1. Die Alpenkrimi-Literatur füllt schon einige Zeit lange Regale in den (nicht nur süddeutschen resp. österreichischen und schweizerischen) Buchhandlungen. Diese Spielart der Antiheimatliteratur (oder handelt es sich vielmehr um die Rückkehr der Heimatliteratur?) treibt mittlerweile besondere Blüten, die nicht unbedingt ins Gewächshaus der traditionellen Antiheimatliteratur gehören, wenn beispielsweise die Romane des Autorenkollektivs Kobr und Klüpfel im von diesen immer wieder inszenierten Allgäu einen eigenen Tourismus hervorbringen, bei dem es darum geht, den Spuren ihres Kommissars Kluftinger zu folgen. Nachdem sich Proteste gegen ein Grab auf dem Friedhof von Altusried erhoben hatten, weil es für eine Romanbzw. Filmfigur errichtet und nach dem Dreh auf Nachfrage einiger Touristen nicht mehr abgebaut worden war, berichtete sogar die Süddeutsche Zeitung über diese Auswüchse des Klufti-Kultes. Vgl. o. V., „Der Tote, der nie existierte“, Süddeutsche Zeitung, 1. August, 2012 
mit ein wenig mehr Tristesse als idyllischen Klischees dekorierten Karren spannt. Auf den zweiten Blick, wenn man die speziellen (Literatur-)Geschichten Revue passieren lässt, wird klar, dass die Antiheimatliteratur als Antipode der Heimatliteratur natürlicherweise ihre Gegenteiligkeit - motiviert durch eine durch die Weltkriege radikal gewandelte historische Hintergrundsituation - ex negativo aus den Merkmalen der in verschiedenen deutschsprachigen Ländern existenten Heimatliteratur bildet und dadurch gleichzeitig eine komplementäre Strömung zu dieser darstellt.

Ein „Katalysator“, der die Transformation des Genres Heimatliteratur zur Antiheimatliteratur ermöglichte, war, wie dieser Beitrag zu zeigen versucht, das Erbe des Naturalismus. Bestimmte thematische und sprachliche Elemente der nach 1945 entstandenen Klischees der Heimatliteratur, bzw. der Blut-und-Boden-Literatur destruierenden Texte sind ohne ein Rekurrieren auf diese Strömung schwer denkbar. Möglichen Spuren des Naturalismus, der bezeichnenderweise in den $1960 \mathrm{er}$ und 1970er Jahren eine Art Revival in der Literaturgeschichtsschreibung erlebt hat - also in der Zeit, in der die Antiheimatliteratur entsteht -, soll in den nachfolgenden Abschnitten nachgespürt werden. ${ }^{3}$

Dazu wurden Beispiele ausgewählt, die als typisch für das Genre der österreichischen Heimatliteratur gelten dürfen: Hans Lebert schuf mit Wolfshaut 1960 einen ebenso richtungweisenden wie kontroversen Text, der in der Nachkriegsliteratur (auch abseits der Antiheimatliteratur) durch die Einführung neuer Motive eine Vorreitertolle spielte. Josef Winklers Ackermann aus Kärnten, 1980 erschienen, kann als ein Roman aus der Hochzeit des Genres betrachtet werden, während Norbert Gstreins Erzählung Einer aus dem Jahr 1988 bereits vom Genre her aus der vom Beitragstitel gesetzten Umzäunung tanzt.

2. Der sogenannte Anti-Heimatroman wurde bereits unter den Begriffen „Neorealismus", oder "kritischer Heimatroman" und "Arbeiterliteratur" beschrieben. Vgl. Ulrike Landmann, Der Anti-Heimatroman - ein österreichisches Phänomen? Zur Entwicklung einer Literaturströmung mit dem Schwerpunkt eines Vergleichs zur Schweizer (Anti-Heimat)Literatur. Diplomarbeit, Universität Wien (PhilologischKulturwissenschaftliche Fakultät), 2012, S. 6.

3. Vgl. Ingo Stöckmann, Naturalismus, Stuttgart, Metzler, 2011., S. 3. 


\section{Überlegungen zum Naturalismus in der österreichischen Antiheimatliteratur}

Um vom impliziten Einfluss des Naturalismus auf die Antiheimatliteratur sprechen zu können, erfordert es - zugegebenermaßen nicht notwendigerweise - erst einmal einen „österreichischen Naturalismus“ zu postulieren; dennoch erscheint ein kurzes Abschweifen zu diesem thematischen Nebenschauplatz insofern interessant, als die Diskussion darum deklarierte naturalistische Elemente in der Antiheimatliteratur weniger "exotisch“ erscheinen lässt, sondern vielmehr als eine „genuine", logische und konsequente Teiltransformation des Naturalismus mehr als 70 Jahre nach seinem eigentlichen Entstehen.

Werner Michler beispielsweise verweist auf die frühere Feststellung des vermeintlichen Fehlens eines Naturalismus in den habsburgischen Ländern, wobei er sich dieser kategorischen Haltung entgegenstellt und die Existenz einer österreichischen Variante desselben von einer differenzierteren Definition abhängig macht. Er verweist auf dessen Charakterisierung als konsequenten Realismus. ${ }^{4}$ Dieser Aspekt wird im Anti-Heimatroman noch auftauchen, wenn es darum geht, die Realität konsequent und schonungslos zu schildern. ${ }^{5}$ Michler konstatiert, der Naturalismus ließe sich

am ehesten also [...] als Syndrom beschreiben, als Syndrom durchaus ungleichnamiger Elemente, aus literarischen Organisationsformen, Schreibweisen, Motiven, Gattungsoptionen, interdiskursiven Anschlüssen, Politiken mit erkennbaren ,Inhalten ${ }^{\text {' }}$ und ,Formen ${ }^{\text {c }}{ }^{6}$

Paradoxerweise verbindet sich das Attribut „österreichisch“ mit dem Naturalismus auch nach einschlägigen Forschungen und Publikationen in den letzten Jahren nach wie vor nur zögerlich, während die

4. Vgl. Werner Michler, „Zur Frage eines österreichischen Naturalismus“, in Roland Innerhofer, Daniela Strigl (Hg.) Sonderweg in Schwarzgelb? Auf der Suche nach einem österreichischen Naturalismus in der Literatur, Innsbruck, Studienverlag, 2016, S. 21-32. (Zitat S. 22).

5. Stöckmann plädiert in diesem Zusammenhang zu Recht für einen differenzierteren Diskurs bzgl. der Realitätsdarstellung, bzw. „Abbildungsanalogien“ im deutschsprachigen Naturalismus, indem er darauf hinweist, dass bei der Aufnahme und Beschreibung der Wirklichkeit dem Realen stets noch ein Transzendentales gegenübergestellt bleibt. Vgl. Ingo Stöckmann, Der Wille zum Willen. Der Naturalismus und die Gründung der literarischen Moderne 1880-1900. Berlin, New York, Walter De Gruyter, 2009, S. 42.

6. Michler, 2016, S. 24. 
Antiheimatliteratur sich diese Zuordnung, wie es scheint, nachhaltig $\mathrm{zu}$ erobern vermochte - abgesehen von gewissen Zugeständnissen (wie z.B. auch die Schweiz habe eine spezifische Antiheimatliteratur hervorgebracht oder auch in der Bundesrepublik sei neuerdings eine solche entstanden). ${ }^{7}$ Eine gängige Erklärung für die kongeniale Kombination zwischen „Österreich“ und dem Antiheimatroman wird bisweilen daraus abgeleitet, dass die rot-weiß-rote Alpenrepublik aufgrund der geographischen Lage (manchmal kleinstädtisch, oft ländlich-dörflich) ${ }^{8}$ als literarischer Schauplatz geradezu prädestiniert sei. Robert Menasse spricht von Österreich als „Anti-Heimat par excellence “9. Theoretisch wären ähnliche Gegebenheiten zwar auch im süddeutschen Raum vorhanden, allerdings spielt hier ein anderer Aspekt mit hinein: Die Frage nach der Vergangenheit und deren nicht erfolgter Bewältigung - sprich: das Erbe des Zweiten Weltkrieges. Während Deutschland eindeutig als das Land der Täter definiert ist, trifft dies für Österreich in dieser Form nicht zu. Ungeachtet der realen Verschränkung hinsichtlich der Machtverhältnisse des Dritten Reiches existierte in Österreich einerseits sehr lange die mittlerweile revidierte Auffassung, das Land sei 1938 Opfer der Nationalsozialisten geworden (Stichwort: „Opfer-Mythos") und andererseits - nach Menasse mit Stand 1996 immer noch - ein weitestgehend tabuisierter, hausgemachter Faschismus:

Der historische Merk- und Leitsatz Österreichs, des Landes, aus dem ich komme, lautet: „Wir haben aus der Geschichte gelernt: Nie wieder Anschluß, nie wieder Faschismus!“ Was ist in diesem Satz verdrängt und vergessen! Etwa, daß Österreich historisch nicht erst einen ,Anschluß brauchte, um faschistisch zu werden. Und welche Möglichkeiten eröffnet diese Zukunft?

7. Die Rezension von Klaus Zeyringer zu Juli Zehs Roman Unterleuten aus dem Jahr 2016 titelt beispielsweise mit „Deutschland hat den Antiheimatroman entdeckt“ und merkt an, dass offensichtlich auch in der Bundesrepublik das „Interesse für abgeschiedene, kaum bedachte Gegenden steigt“. Klaus Zeyringer, „Juli Zeh: Deutschland hat den Antiheimatroman entdeckt“, Der Standard, 17. März 2016, Zugriff am 11.2.2017, von URL: http://derstandard.at/2000032730469/Juli-Zeh-Deutschlandhat-den-Antiheimatroman-entdeckt.

8. Vgl. Josef Donnenberg, „Heimatliteratur in Österreich nach 1945 - rehabilitiert oder antiquiert?", in Karl Konrad Polheim (Hg.), Wesen und Wandel der Heimatliteratur: Am Beispiel der österreichischen Literatur seit 1945, Bern [u.a.], Peter Lang, 1989, S. 39-68 (Zitat S. 42).

9. Robert Menasse, Hysterien und andere historische Irrtümer. Mit einem Nachwort von Rüdiger Wischenbart, Wien, Sonderzahl,1996, S. 28. 
Zumindest die, daß wir keinen Faschismus als ,faschistisch' bezeichnen können oder müssen, der die staatliche Souveränität unangetastet läßt. ${ }^{10}$

Eine Mitverantwortung (und Vorbelastung) konnte daher lange erfolgreich ausgeschlossen werden. ${ }^{11}$ Anfang der 1960er-Jahre erst machte sich in Österreich die Tendenz bemerkbar, ,sich kritisch-kreativ mit den Themen und Motiven der traditionellen Heimatliteratur auseinanderzusetzen". ${ }^{12}$ Müller-Funk spricht kritisch von einer

Selbstbildlichkeit und Autostereotypie [...], der sich die österreichische Literatur insbesondere nach 1968 unterworfen hat. Die Rede ist vom Etikett der Antiheimatliteratur, dem problematisierten Heimatroman, einem Phänomen, das auf geniale wie prekäre Weise zwei Momente österreichischer Befindlichkeit und symbolischer Gestaltung miteinander verbunden hat: eine rhetorisch aufgeladene Kritik an der unseligen Verquickung des eigenen Landes mit der nationalsozialistischen Vergangenheit und eine Hinwendung $\mathrm{zu}$ jenen ländlichen Peripherien, die [...] als realer und symbolischer Ort dieser Symbiose von Österreichertum und Nationalsozialismus angesehen wurden $[\ldots] .^{13}$

Die Austriazität der Antiheimatliteratur wird sogar als ein integraler definitorischer Bestandteil wahrgenommen, was ungeahnte Folgen nach sich ziehen könnte, wie Müller-Funk polemisch anmerkt, wenn er 2010 die Frage der Zukunft des Antiheimatromans stellt:

Wenn also Heimat und Anti-Heimat in der politischen Rhetorik und im literarischen Leben spürbar zurücktreten, wenn 50 Jahre nach dem Tod Heimito von Doderers die Stadt als heterogener und polyphoner Raum ins Zentrum des Interesses rückt, dann muss man sich die Frage vorlegen, was das für Konsequenzen für eine spezifisch österreichische Literatur hat und ob nicht mit dem Auslaufen der Antiheimatliteratur ein entscheidendes Moment der Literatura austriaca abhandenzukommen droht. ${ }^{14}$

Eine erste Brücke zwischen den auf den ersten Blick disparaten Phänomenen des Naturalismus und der Antiheimatliteratur ergibt sich, um

10. Ebd.

11. Klaus Amann, Der Zweite Weltkrieg in der Literatur. Österreichische Beispiele, Bregenz, Erinnern.at, 2002, S. 21.

12. Andrea Kunne, „Heimatromane Postmodern. Zur Transformation einer Gattung am Beispiel von Reinhard P. Gruber, Gert Jonke und Max Moetz", in Hubert Orlowski (Hg.), Heimat und Heimatliteratur in Vergangenheit und Gegenwart, Poznan, Instytut Filologi Germanskiej Uam 1993, S. 101-115 (Zitat S. 102).

13. Wolfgang Müller-Funk, „Ein Koffer namens Österreich“, Die Presse, 15. Jänner 2010. Zugriff am 27.09.2016, von http://diepresse.com/home/spectrum/ zeichenderzeit/533178/print.do.

14. Ebd. 
im Bereich historischer Überlegungen zu bleiben, eventuell bereits aus der sie generierenden geschichtlichen Dynamik: Wie der Naturalismus entwickelt sich die Antiheimatliteratur auch in Reaktion auf eine tiefgreifende historische und gesellschaftliche Veränderung (siehe z.B. die Aussagen Müller-Funks). Die industrielle Revolution verursachte nachhaltige Veränderungen im Deutschen Reich und - mit einer gewissen zeitlichen Verzögerung - auch in der Donaumonarchie. Dieser Prozess beschränkte sich natürlich nicht nur darauf, dass neue urbane Welten entstanden, sondern auch Eingriffe in die ländliche Sphäre (wie z.B. die sukzessive Technisierung, der Ausbau des Tourismus usw.) ihre Spuren hinterließen. Kurz: eine politische und ästhetische bzw. literarische Reaktion wurde unaufschieb- und unabdingbar, in deren Zentrum die Auseinandersetzung mit der neuen Realität stehen musste. ${ }^{15}$ Die Heimatliteratur kann in diesem Sinne als ein Aufbäumen gegen diesen innovativen Impetus gesehen werden. Innerhalb des wirklichkeitsorientierten, naturalistischen Konfrontationsprozesses wurde bekanntermaßen speziell die exakte Beschreibung von Mensch und Umwelt sowie deren Beziehungen mit- und untereinander wesentlich drastischer und exakter betrieben als noch im „bürgerlichen Realismus“ und der „Gründerzeitliteratur". ${ }^{16}$

Die Notwendigkeit einer Auseinandersetzung mit einer behaupteten Wirklichkeit, „einer irreell heilen Welt ${ }^{\text {“17 }}$, die in Österreich nach dem Zweiten Weltkrieg, wie Menasse feststellt, geradezu museal inszeniert worden war, verbindet die beiden Strömungen zumindest auf der Ebene der literarischen Zielsetzung und des literarischen Impetus. Während es im traditionellen Heimatroman des 19. Jahrhunderts, der aufgrund der „thematische[n] Übereinstimmung mit den völkischen Idealen“ 18 ohne größere Anpassungsnotwendigkeiten von den Nationalsozialisten propagandistisch instrumentalisiert werden konnte, darum gegangen war, positive bäuerliche Welten zu entwerfen, machte sich die Antiheimatliteratur an die Aufdeckung der Tristesse und der Hässlichkeit im ländlichen Kosmos inklusive seiner Bewohner und derer zumeist als dysfunktional und pathologisch beschriebenen Beziehungen.

15. Erhard Schütz, Jochen Vogt, Einführung in die deutsche Literatur des 20. Jahrhunderts. Band 1: Kaiserreich, Opladen, Westdeutscher Verlag, 1977, S. 28.

16. Ebd.

17. Ingeborg Rabenstein-Michel, „Bewältigungsinstrument Antiheimatliteratur“, Germanica. Vol. 42. (2008). Zugriff am 24.1.2017, von URL: https://germanica.revues.org/525?lang=de, S. 1-16 (Zitat S. 4).

18. Kunne, op. cit., 1993, S. 102. 
Heimatroman vs. Anti-Heimatroman:

Naturalistische Berührungspunkte

Bei Wilhelm Solms dagegen taucht der Gedanke auf, dass Heimatund Antiheimatliteratur womöglich nur zwei Seiten derselben Medaille bilden: Dies wäre eine Literatur, „die sich nicht gegen Heimat, sondern gegen traditionelle Heimatliteratur wendet und die damit womöglich zu einem neuen Verhältnis zur Heimat beiträgt. " 19

Die Berührungspunkte werden jedoch nicht ausschließlich zwischen der Literatur des Naturalismus und der (Anti-)Heimatliteratur festgestellt, sondern können innerhalb einer langen literarischen Tradition gesehen werden. Eugen Thurnher geht soweit, die Ursprünge des Heimatromans bereits bei Homer und der Heimkehr des Odysseus anklingen zu sehen, auch wenn er den Heimatroman nicht als unmittelbaren Nachfolger der antiken Dichtung sieht. ${ }^{20}$ Als erstes deutschsprachiges Beispiel für einen Heimatroman macht er den um etwa 1250 entstandenen Meier Helmbrecht aus, mit einer interessanten Pointe, die thematisch den Bogen zum Antiheimatroman schlägt, indem sie eines seiner „klassischen“ Merkmale benennt: Im Mittelpunkt steht neben der (auch für den Heimatroman) obligaten dörflichen Welt ein junger Mann in der Rolle eines Anti-Helden. ${ }^{21}$ Auch den naturalistischen Texten geht es vom Personal her gesehen um diejenigen, die versuchen in einer feindlichen Lebenswelt ihren Platz zu finden oder zu behaupten.

Ein weiteres Kriterium ist die Perspektive, aus der erzählt wird. Nach Zeyringer und Gollner steht im Heimatroman die Perspektive des Bauern im Vordergrund, während im Anti-Heimatroman (und in der Regel auch im Naturalismus) jene „der Knechte, Mägde, Lehrlinge und Akkordarbeiter“22 dominiert. Dabei treten unterschiedlichste „Pathologien“ zutage, die sowohl die Umgebung, die Physis und Psyche der

19. Wilhelm Solms, „Zum Wandel der ,Antiheimatliteratur““, in Karl Konrad Polheim (Hg.), Wesen und Wandel der Heimatliteratur: Am Beispiel der österreichischen Literatur seit 1945, op. cit., S. 173-189 (Zitat S. 173).

20. Vgl. Eugen Thurnher, „Plädoyer für den Heimatroman”, in Karl Konrad Polheim (Hg.), op. cit., S. 5-37 (Zitat S. 37).

21. Vgl. ebd., S. 27.

22. Klaus Zeyringer, Helmut Gollner, Eine Literaturgeschichte: Österreich seit 1650, Innsbruck [u.a.], Studien Verlag, 2012, S. 674. 
Protagonisten, wie auch die sozialen Umstände betreffen. ${ }^{23}$ Die Grundstimmung ist eine fortgesetzt gestörte, unharmonische, das ehemals gültige Wertesystem nicht mit der veränderten Gegenwart vereinbar und brüchig geworden. Es gilt das Gegenteil von dem, was Thurnher für den Heimatroman konstatiert: „Heimat ist nicht unbedingt ein bestimmter Ort, Heimat ist ein innerer Zustand, sie bedeutet das Glück der Übereinstimmung von Welt und Ich. Heimat ist überall. “24 Daraus folgert er, dass , der Heimatroman in einem Kontext gesehen werden muß, der zwar die Grade und Unterschiede nicht auslöscht, aber die Gleichheit des Wesens und des Wollens betont. ${ }^{25}$ In diesem Sinne kann wiederum, in Abgrenzung zu dieser Definition, für den Naturalismus (und auch für die Antiheimatliteratur) behauptet werden: Hier wird eben gerade der Verlust bzw. das Nichtvorhandensein einer Kongruenz zwischen Ich und Dorf, Ich und traditionellem Wertekosmos, beschrieben, wobei für krude Realitäten auch eine krude, oft dialektal geprägte Sprache verwendet wird. Die Heimatlosigkeit als ein Merkmal der Literatur der Moderne spiegelt sich auch in den literarischen Kämpfen der Antiheimat-Autoren wider. Wilhelm Solms bezeichnet diese Form der Literatur als „,radikale Antiheimatliteratur" ${ }^{26}$ Der Antiheld, der sich darin bewegt, findet die Heimat weder um sich herum noch in sich selbst noch in den gültigen gesellschaftlichen Wertesystemen. Dabei beschränkt sich die Darstellung nicht auf die Beschreibung des „sozialen Milieus Dorf", sondern versucht, die mimetische Ebene durchaus mit korrespondierenden symbolizistischen Bedeutungsdimensionen zu verbinden - und nähert sich darin wiederum dem Naturalismus; ${ }^{27}$ dies wird insbesondere bei der Auseinandersetzung mit den ausgewählten Textbeispielen, speziell mit Wolfshaut, deutlich.

23. Das Interesse für Krankheit erweist sich aber im Naturalismus (wie in der Antiheimatliteratur) nicht als ein einseitiges, wie Stöckmann anmerkt: Krankheit kann sowohl Gegenstand von Tabuisierung als auch Gegenstand wissenschaftlicher Neugier sein. Vgl. Stöckmann, 2011, S. 52.

24. Thurnher, „Plädoyer ...“, in K. K. Polheim (Hg.), op. cit., 1989, S. 28.

25. Ebd., S. 37.

26. Solms, „Zum Wandel ...“, in K. K. Polheim (Hg.), op. cit., 1989, S. 173.

27. Vgl. Stöckmann, Naturalismus, op. cit., 2011, S. 110. 


\section{Außenseiter, Unheim(at)liches und Vernichtung:} Ausgewählte Beispiele

\section{Hans Lebert - Wolfshaut}

Hans Leberts Roman Wolfshaut erschien, wie bereits eingangs erwähnt, im Jahr 1960, also vor der Hochphase des Anti-Heimatromans, die ab der Mitte der $1970 e r$ Jahre begann. ${ }^{28}$ Er steht damit „am Anfang der Entwicklung in der österreichischen Nachkriegsliteratur, in der engagierte Autoren bestrebt sind, sich vor allem von einem kritischen Standpunkt aus mit dem Thema ,Heimat' auseinanderzusetzen ". ${ }^{29}$ Somit gilt Wolfshaut als „einer der ersten Romane, in denen das traditionelle Genre eine Wiederaufnahme in den literarischen Kanon erfährt“ ${ }^{*}{ }^{30}$ Gleichzeitig bedeutete diese Wiederaufnahme jedoch eine Transformation, die sich in gewissem Maße im Rahmen der oben beschriebenen „naturalistischen“ Mechanismen begreifen lässt. Die zentralen Merkmale des Heimatromans fallen jedoch weg, beziehungsweise werden „kritisch" umgewandelt und im Sinne einer klischeebefreiten Bestandsaufnahme instrumentalisiert: Weder der Glaube, noch die Landschaft, noch die Menschen bieten Zuflucht:

Der Heimat im traditionellen Sinn wird in den Texten Leberts ein anderes Konzept gegenübergestellt. Die Heimat, von der die Protagonisten sprechen, kommt den Figuren als Wunschvorstellung zu. Der Geschmack und der Geruch bieten die Möglichkeit, Heimat sinnlich zu vermitteln. Zudem knüpfen sich Bilder der Ferne, wie Gebirge und Meer, an die Vorstellung von Heimat. Die Lebertschen Figuren sind nicht in der Heimat, sie sind bestenfalls auf dem Weg dahin. ${ }^{31}$

„Es ist eine gottverlassene Gegend, eine Gegend, die nichts zu bieten hat und deshalb auch kaum bekannt ist. “" ${ }^{32}$ So charakterisiert der Erzähler zu Beginn des Romans den Ort der Handlung. Jürgen Egyptien konstatiert, die im Roman herrschenden Witterungsverhältnisse seien „an Trostlosigkeit und Ingrimm [...] schwer zu übertreffen " ${ }^{33}$ Die Schilderung der

28. Vgl. Zeyringer/Gollner, op. cit., S. 673 .

29. Kunne, op. cit., S. 105.

30. Ebd., S. 103.

31. Florian Braitenhaller, Küss mich, du Schwein! Hans Leberts diskrete Beziehung zur Moderne, Wien, WUV Universitätsverlag, 2003, S. 146.

32. Lebert, Wolfshaut, S. 7.

33. Jürgen Egyptien, „Kreuzfahrten durch den leeren Himmel. Hans Leberts ,Wolfshaut' als transzendentales Logbuch" in Hans Lebert, Die Wolfshaut, Wien [u.a.], Europaverlag, 1993, Nachwort S. 599-627 (Zitat S. 599). 
äußeren Verhältnisse (Wetter, Natur) steht aber in einem inneren Bezug zur Handlung und spiegelt somit das Geschehen wider. „Zwischen der deskriptiven Naturschilderung und ihrer symbolischen Aufladung steht das Reich der Metapher, das bei Lebert von einer einzigartigen Eindringlichkeit und bei aller Konzentration aufs Verhängnis unerschöpflichen Fülle ist. “34 Während die Schweigsamkeit („Schweigen“ heißt in Leberts Roman der Ort, in dem das Geschehen spielt) im traditionellen Heimatroman mit positiven Konnotationen verbunden ist ${ }^{35}$, wird das Schweigen bei Lebert negativ besetzt. Schweigen bezieht sich hier auf eine verdrängte Vergangenheit und bedeutet demnach Verschweigen. Stilistisch gelingt es Lebert, „bewährte Motive und Strukturen des traditionellen Heimatromans kontrapunktisch zu zitieren ". ${ }^{36}$ Deutlich wird dies im sprachlichen Ausdruck. Die formale Beschreibung natürlicher Elemente mischt sich jedoch mit drastischen inhaltlichen Schilderungen des Geschehens: „[...] flammte an einem glasklaren und veilchenblauen Horizont [...] bei einer Kälte, daß die Gehirnschale krachte -, die Sonne wie ein frischer Blutfleck auf. "37 Solche Beispiele finden sich mehrfach im Roman: „[...] der Höhe ziemlich nahe, aus welcher der Himmel, zerschlagen von hellblondem Licht, in blauen Scherben auf sie niederprasselte. ${ }^{\text {“38 }}$ Besonders deutlich wird die Verwendung dieser Technik, wenn die Schilderung der Natur mit einem Körper und - in letzter, drastischer Konsequenz - mit einem Schlachthaus assoziiert wird:

Eine hohe, dünne Wolkenschicht, einem Netz von feinen Adern gleichend, war - vermutlich schon vor dem Sonnenuntergang - über das blaue Gewölbe des Himmels gewachsen. Während wir in ungestillter Jagdgier einem Schatten nachgelaufen waren, hatte eine unsichtbare Spinne ein Gewebe aus Blutadern über den Himmel gesponnen. Nun waren die Adern geplatzt, und das Blut quoll hervor und fiel als roter Regen auf uns nieder; alles ward rot, die Straße, der Schnee, das Gebirge, überflutet wie in einem Schlachthaus. ${ }^{39}$

34. Ebd.

35. Vgl. Kunne, op. cit.,, S. 103.

36. Rossbacher, zitiert nach Andrea Kunne, Heimat im Roman: Last oder Lust? Transformation eines Genres in der österreichischen Nachkriegsliteratur, Amsterdam, Atlanta, Rodopi, 1991 S. 106.

37. Lebert, Wolfshaut, S. 310.

38. Ebd., S. 323.

39. Ebd., S. 355. 
Immer wieder tauchen derart expressive, beinahe dionysisch anmutende Metaphern auf, die Beschreibung der Natur verweist zugleich konstant auf andere Bedeutungsebenen. Dadurch:

erschöpfen sich nicht einzelne Phänomene in der symbolischen Evokation einer allgemeinen Bedeutungsebene, welche den Handlungsverlauf natursprachlich kommentiert; die Naturgewalten treten selbst in personam gestaltend auf den Plan und überziehen die Welt mit einem Geflecht von Zeichen, das sich zu einem geheimen Logbuch der Geschehensabläufe zusammensetzt. $^{40}$

Die Schilderung der Landschaft vermischt sich dabei auch mit Beschreibungen, die eine verdrängte politische Vergangenheit zitieren:

Vom Winde angetrieben [...] gelangte Maletta ans südliche Ende der Ortschaft und wankte in eine parteibraune Landschaft hinaus. Die Bäume längs der Fahrbahn standen Spalier und hoben grüßend ihre Hände hoch; in weißen Stutzen marschierten die Prellsteine auf; die Windstöße knatterten wie in Standarten und Fahnen. ${ }^{41}$

Für Kunne bildet Wolfshaut „ein besonderes Beispiel für transformierte Heimatliteratur “42, da der Roman mit der Behandlung der Kriegsvergangenheit in einem österreichischen Dorf der Gattung ein neues Element hinzufügt.

\section{Josef Winkler - Der Ackermann aus Kärnten}

Sprachgewalt spielt auch in der Wahrnehmung der Texte von Josef Winkler eine bedeutende Rolle. Aus dessen Feder stammen nach Schnell „die sprachmächtigsten Zeugnisse einer autobiographisch motivierten Literatur [...] im Übergang zu den achtziger Jahren". ${ }^{43}$ Der Roman Der Ackermann aus Kärnten ist Teil einer Trilogie mit dem Titel Das wilde Kärnten. Es geht um die „Initiation eines homosexuellen Jugendlichen in einer patriarchalisch-dörflichen Welt $[\ldots]{ }^{*} .{ }^{44}$ Dabei fließen

40. Egyptien, „Kreuzfahrten“, in Lebert, op. cit., S. 600.

41. Lebert, Wolfshaut, S. 185.

42. Kunne, Heimat im Roman..., op. cit., 1991, S. 130.

43. Ralf Schnell, „Von 1945 bis zur Gegenwart“, in Volker Meid (Hg.), Geschichte des deutschsprachigen Romans, Stuttgart, Reclam, 2013, S. 688.

44. Hermann Korte, Österreichische Literatur der Gegenwart, Stuttgart, Metzler, 2016, S. 146. 
autobiografische Elemente ein. ${ }^{45}$ Winkler bricht Tabus und kreist immer wieder „um wenige sich wiederholende Motiv- und Stoffpartikel zu den beherrschenden Polen Sexualität und Tod. “46 Die Schilderung des Dorfes wird mit Kapitelüberschriften dramatisch inszeniert, beziehungsweise „annonciert“, indem jedes einzelne Haus einer Klassifizierung unterzogen wird: „Haus 1 Kein Tier, 3 Kinder, 7 Kruzifixe“. ${ }^{47}$ Das Geschehen in der bäuerlichen Umgebung, wie zum Beispiel das Sterben eines Kindes bei der Geburt, wird lapidar, doch semantisch und "landschaftlich aufgeladen" geschildert:

Die Fensterkreuze ließen den Blick bis ans Ende der Welt gehen. Jedes Auge, das aus dem Fenster blickte, durchdrang die Landschaft, die grünen Wiesen, der Wind schlug Wogen im Roggenfeld, millionenfach zitterten die Haferähren ums tote Kind. ${ }^{48}$

Die Tristesse der Existenz in dieser „Anti-Heimat“, in der das Kreuz immer wieder den Blick verstellt, spiegelt sich auch in dem dadurch (und durch die unausgesprochene Vergangenheit voller großer und kleiner Traumata) pathologisierten Beziehungsgeflecht wieder. Homosexualität hat darin keinen Platz und soll in einen toten Winkel abgeschoben bleiben. Der Autor verarbeitet dieses in der Heimatliteratur tabuisierte Thema in Form einer äußerst dichten, expressiven, farbigen und plastischen Sprache, die sich gleichermaßen als Ausdruck und Bewältigungsversuch einer familiären und regionalen Konstellation des Leidens und der Unterdrückung, des Begehrens und des Todes verstehen lässt. ${ }^{49}$ Christina Ujma beschreibt diese spezielle Art des Ausdrucks in der

45. Heydemann spricht in seiner Untersuchung zu Ebner-Eschenbachs Das Gemeindekind, Waggerls Das Jahr des Herrn und Innerhofers Schöne Tage von Erklärungsmöglichkeiten, die sich aus der „Herkunft der Autoren“ ergeben würden, die zu den geschilderten Ereignissen „ein besonderes Nahverhältnis“ aufweisen würden; von einem Nahverhältnis, um nicht von autobiographischen Zügen zu sprechen, kann - in der Regel - auch in Zusammenhang mit den Texten der Antiheimatliteratur geredet werden, wobei zumeist mindestens eine geographische Verbindung zum Ort des Geschehens besteht. Vgl. Klaus Heydemann, „Jugend auf dem Lande. Zur Tradition des Heimatromans in Österreich“, in Friedrich Aspetsberger (Hg.), Traditionen in der neueren österreichischen Literatur, Wien, Österreichischer Bundesverlag, 1980, S. 83-98 (Zitat S. 95).

46. Korte, Österreichische Literatur..., op. cit., S. 146.

47. Josef Winkler, Der Ackermann aus Kärnten. Roman, Frankfurt am Main, Suhrkamp, 1984, S. 45 (zitiert wird nach dieser Ausgabe).

48. Ebd., S. 52.

49. Vgl. Schnell, „Von 1945 ...“, in Meid, op. cit., 2013, S. 688. 
Novelle Natura morta und kommt zu einem Schluss, der sich in Teilen auch auf den Ackermann aus Kärnten übertragen ließe:

Es ist die poetische Eindringlichkeit von Winklers Sprache, die sowohl den Markt als auch Piccoletto zum Leuchten bringt. Seine Beschreibungen sind von barocker Opulenz, aber auch von einer Exaktheit und Genauigkeit, die beinah naturalistisch anmutet. ${ }^{50}$

Doch dient diese Opulenz keinem Selbstzweck, sondern gleichzeitig der Skizzierung und Interpretation des Milieus, wie Korte feststellt:

Die poetische Zustandsmetapher des ersten Bandes konkretisiert sich im Ackermann aus Kärnten zu einem kritischen Soziogramm des Dorfes; [hier] versucht Winkler, mit den Mitteln der Ethnopoesie die Alltags- und Festrituale des Dorfes zu entschlüsseln. ${ }^{51}$

Das ist ein weiterer Hinweis auf einen Anti-Heimatroman, weil der klassische Heimatroman solche Szenen zwar schildert, ohne sie jedoch kritisch zu entschlüsseln. Das beinahe wissenschaftliche, gnadenlos enthüllende Interesse und dessen thematische Stoßrichtungen (Sexualität, Tod, Gruppendynamik, Ritualität usw.) verbinden den Autor mit den Traditionen der Moderne, und speziell mit dem Naturalismus; akzentuiert wird im Rahmen dieses Impetus' auch die Kirchenkritik, beziehungsweise die Kritik am Katholizismus im Speziellen, ganz im Sinne der österreichischen „Nestbeschmutzer“-AutorInnen im Umkreis der Antiheimatliteratur: „Die geographische Anatomie unseres Dorfes lässt sich mit einem Kruzifix vergleichen. " 52 Der für die Heimat so bedeutende Katholizismus taucht auf, ebenso wie ein diffuser Stolz auf die Heimat, der sich aber nur gebrochen zeigen kann, assoziiert mit einem Versehrten: „Stolz auf Kärnten war ich, als ich im Vergnügungspark der Klagenfurter Holzmesse einen Invaliden im Kärntneranzug sah. “53 Wortmächtige Sätze wie folgender erweisen sich als typisches Stilmittel und als besondere Strategie, Natur und ethische Wertung miteinander zu verbinden: „Das Dorf reduziert sich zu einem faulen Getreidekorn. “54 Natur wird gleich in mehreren Dimensionen zur Bedeutungsträgerin,

50. Christina Ujma, „Barocker Naturalismus. Josef Winklers römische Novelle ,Natura Morta“", literaturkritik.de, Nr. 4. April 2002. Zugriff am 12.2.2017, von URL: http://literaturkritik.de/public/rezension.php?rez_id=4849\&ausgabe=200204, S. 1-3 (Zitat S. 2).

51. Korte, Österreichische Literatur, op. cit., 2016, S. 146.

52. Winkler, Der Ackermann ..., S. 10.

53. Ebd., S. 270.

54. Ebd., S. 276. 
als ebenso typische Charakteristika der Darstellung erweisen sich Anspielungen, in denen sich das Empfinden des Erzählers mit Begriffen aus der Pflanzenwelt vermischt: „Die Altäre meiner Kindheit sind mit Moos überwachsen, manchmal wischt eine kindliche Handbewegung es zur Seite und stellt einen Kelch darauf. “55

\section{Norbert Gstrein - Einer}

Die Einordnung Norbert Gstreins in die Antiheimatliteratur begründet sich darin, dass seine ersten fünf Bücher inhaltliche Bezüge zu Tirol aufweisen. ${ }^{56}$ „In seiner ersten Erzählung Einer (1988) stellt er den Zerfall der Gemeinschaft im Fremdenverkehrsort dar, konzentriert am Beispiel eines zerstörten Individuums, des Außenseiters Jakob. “57 Das Dorf in seiner Erzählung ist - ganz in der Tradition der Antiheimatliteratur eben kein trauter Ort, die Heimat scheint längst verloren, sie wird und ist wirtschaftlich ausgeschlachtet: „In schamloser Oberflächlichkeit versuchten sie, die Leute im Dorf nachzuahmen, und ahnten nicht, daß längst nichts mehr stimmte und alles verkauft war für billiges Geld. “58 In seiner Erzählung Einer kommt der junge Mann, wieder ein AntiHeld, selbst nicht zu Wort. Er bleibt sprachlos und setzt damit einen

55. Ebd., S. 277.

56. Die Frage, wie die Autoren, im Speziellen die hier genannten, zu ihrer Klassifikation als Vertreter der Antiheimatliteratur stehen, kann an dieser Stelle nicht ausführlicher behandelt werden; Lebert selbst verweigerte sich Zuordnungen dieser Art, und Gstrein entwindet sich dieser Klassifikation auch weitestgehend, wobei er von der Literaturwissenschaft auch argumentative Unterstützung erfährt. Es scheint fast, dass das Etikett „Antiheimatliteratur“ ein unerwünschtes sei, bzw. als limitierend wahrgenommen wurde und wird; für alle drei in diesem Artikel angeführten Texte gilt, womit Gunreben ihre Überlegung schließt (und was deren Klassifizierung als der Antiheimatliteratur zugehörige Werke in Frage stellen muss): „Diese Themenwahl könnte Gstrein der seit Thomas Bernhard zum Schlagwort gewordenen österreichischen Antiheimatliteratur stempeln, hätte er sie nicht, und auch das wurde in der Kritik vielfach betont, eigenwillig umgesetzt: Verschiedene Erzählungen sind ineinander verschachtelt, die Perspektiven wechseln häufig, oft ist nicht eindeutig zu bestimmen, welche der Figuren überhaupt spricht. Der formale Anspruch dieser Texte und ihre komplexe Sprache brachten viele Kritiker zu der Überzeugung, nicht das Thema mache den Rang der Erzählung aus, sondern die Form und die Sprache." (Marie Gunreben, Der Literatur mit ihren eigenen Mitteln entkommen. Norbert Gstreins Poetik der Skepsis, Bamberg, University of Bamberg Press, 2011, S. 7)

57. Zeyringer/Gollner, op. cit., S. 746.

58. Norbert Gstrein, Einer, Frankfurt am Main, Suhrkamp, 1992, S. 56 (zitiert wird nach dieser Ausgabe). 
Merkmalstrang fort, der bereits in Wolfshaut und im Ackermann aus Kärnten relevant war. Erzählt wird die Geschichte aus unterschiedlichen Perspektiven. Der Protagonist Jakob ist durch traumatische Erlebnisse in der Stadt gezeichnet. Nach seiner Internatszeit dort vermag er sich nicht mehr im Dorfleben einzufinden. „Erinnerungen. Aber was erklären sie, wenn man nicht ein Träumer ist oder wie das Dorf, das im Nachhinein leichtfertig alles weiß?" ${ }^{59}$ Die Hoffnungslosigkeit des Protagonisten Jakob deutet sich in den Metaphern (Tag/Nacht, hell/dunkel) an: „Die Nächte nähmen zu, und in ein paar Wochen würde die Sonne auf ihrem Weg nicht mehr über die höchsten Gipfel kommen und schon um zwei, früh am Nachmittag, untergehen. “60 In düsteren Worten wird die in der klassischen Heimatliteratur gottgegebene und gottgewollte (positive) Beziehung zwischen Natur und Mensch geschildert; die intakte Natur gibt es aber nicht mehr, sie ist "versehrt" und verweigert sich den Wünschen der Menschen:

Im Jänner blieb der Schnee aus, die Pisten wurden scheckig von den aperen Stellen, die unter der dünnen Schicht hervorkamen, und man beschwichtigte stets von neuem die Gäste, die abzureisen drohten, versprach ihnen das Weiße vom Himmel und wußte nicht, in welchen Worten die schlechte Saison noch zu beklagen wäre, weil es keine mehr gab. ${ }^{61}$

Die äußeren Umstände in Form der Naturgewalten, wie das Wetter, konditionieren zwar immer noch die (Überlebens)- und Zukunftschancen der Menschen, sie werden aber auch von der Industrialisierung (vom Tourismus) in die Mangel genommen. Die Beziehung wird vom Kampf zum „dreckigen Geschäft“. Für den Protagonisten Jakob wird das, was von der Natur bleibt, zum Rückzugsort vor den Menschen; er wählt das kleinere Übel, die überschaubarere Gefahr:

Tagsüber ging er mitunter aus dem Haus, streifte durch den tiefverschneiten Wald, wo es keine Spuren gab, oder saß an seinem Lieblingsplatz in der Sonne und kehrte bei Einbruch der Dunkelheit zurück, schlich in die Kammer und ließ sich nicht mehr sehen bis zum Frühstück am nächsten Morgen. ${ }^{62}$

Die Ambivalenz (und nicht die Eindeutigkeit der Idylle) kennzeichnet die gesamte Umgebung. Für Jakob ist die Heimat ein Ort, der Vertrautes, aber auch Schreckliches birgt. Auch hier ist das Dorf voller mehr oder

59. Ebd., S. 12.

60. Ebd., S. 7.

61. Ebd., S. 30.

62. Ebd. 
weniger dunkler Geheimnisse, von Verbrechen, Tod - und Komplizenschaft:

Die Spaziergänge führten stets zur Kirche, und auf dem Friedhof zeigte er ihnen das Grab des Vaters oder erzählte von dem Mädchen, das ein Kind austrug und allein mit dem Liebhaber zur Welt brachte, vor den Eltern, den Schwestern, dem ganzen Dorf verborgen, und nach der Geburt hätten sie es erschlagen und dort, genau an dieser Stelle, verscharrt. ${ }^{63}$

Die Heimat wird, wie gesagt, vom Tourismus bestimmt, der die dörflichen Rituale ad absurdum führt, die Identität zu Markte getragen - alles wird fake: „Sie fielen nicht aus der zugedachten Rolle, keiner, waren den Gästen zu Gefallen und animierten, animierten oft an drei Tischen gleichzeitig, animierten sich zu Tode." ${ }^{64}$ Heimat wird letztlich dadurch definiert, dass Fremde anwesend sind, als Touristen ${ }^{65}$ auftretend, die durch ihre bloße Anwesenheit und Erwartungen das Leben der Einheimischen bestimmen:

$[\ldots]$ wer weiß noch um das Weinen und Händeringen an den langen Herbstabenden, wenn sie untätig in den leeren Häusern saßen, ausgezehrt vom vergangenen Sommer und in Gedanken, die um Alltäglichkeiten kreisten oder leer waren oder längst im nächsten Winter, wenn das Leben weiterginge und sie hervorholte und wieder zurückstieße in dieselbe Einsamkeit oder eine tiefere? ${ }^{66}$

Es scheint sich bei Gstrein nicht nur die Natur in einer Agonie zu befinden, sondern auch die Menschen, die die marode Kulisse bespielen und nicht mehr wissen, was sie eigentlich ausmacht - die Werte sind nicht nur nicht mehr vorhanden, sogar ihr Vorhandensein in der Vergangenheit wird bezweifelt, beziehungsweise kann nicht mehr erinnert werden:

[...] das Heu von den Steinen, ein Mädchen aus den Bergen und Fleisch von den Knochen, im Dialekt ein gelungener Reim, glaubten sie selbst nicht

63. Ebd., S. 41.

64. Ebd., S. 58.

65. Gebhard, Geisler und Schröter verweisen in ihrem Aufsatz „Heimatdenken: Konjunkturen und Konturen“ auf eine weitere mögliche Verquickung von Heimatlichem und Exotischem, wenn etwa - im Sinne einer Globalisierung - vom Transfer von Folkloregruppen an die verschiedenste Zielorte die Rede ist. Der Export von Heimat erzeugt demnach eine Art „Binnenexotik“. Vgl. Günter Gebhard, Oliver Geisler, Steffen Schröter, „Heimatdenken: Konjunkturen und Konturen. Statt einer Einleitung", in dieselben (Hg.), Heimatdenken: Konturen und Konjunkturen eines umstrittenen Konzepts, Bielefeld, transcript Verlag, 2007, S. 9-56 (Zitat S. 39-40).

66. Gstrein, 1992, S. 58. 
daran oder dachten mit glänzenden Augen an die gute alte Zeit, von der sie wußten, oder wußten sie nicht, daß es sie nicht gab, nie gegeben hat und die Leute immer schon aus dem Vollen schöpfen wollten und am liebsten alles haben und noch mehr? ${ }^{67}$

Jakob ist heimatlos. Jegliche Identifikationsgrundlage ist ihm abhandengekommen. Vor dem Hintergrund des allgemeinen Wahnsinns wird er letztlich als psychisch Gestörter diagnostiziert: „Es war etwas, das er nicht im Griff hatte. Verrücktsein nannte man es im Dorf, nie Krankheit, ein Wort aus einer ganz anderen Wirklichkeit, über die niemand

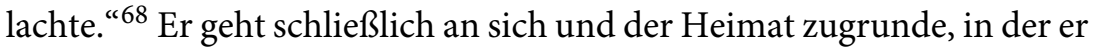
sich nicht mehr zuhause fühlen kann.

\section{Fazit}

Der (österreichische) Naturalismus hat seine Spuren in der Nachkriegsliteratur hinterlassen. Die AutorInnen der Antiheimatliteratur behandeln Themen und bedienen sich Techniken, die durchaus vom Erbe des Naturalismus abgeleitet werden können, wie die kurzen Anmerkungen zu den drei behandelten Werken zeigen sollten. Speziell die österreichische Antiheimatliteratur verfolgt in ihren Texten das Ziel, die nach 1945 behaupteten Klischees der intakten Heimat, beziehungsweise deren intakter, vom Nationalsozialismus unbelasteten Geschichte aufzubrechen. Diese Klischees und Kulissen waren in der Heimatliteratur des 19. Jahrhunderts sowie in der Blut-und-Boden-Literatur, festgeschrieben und perfektioniert worden. Es erscheint daher als folgerichtig, dass die AutorInnen deren zentrale Merkmale aufgreifen und - oft durch Karikierung - destruieren wollten. Das Dorf, das Ländliche, die Natur und die Bewohner mit ihren vom Glauben geprägten, tradierten Ritualen stehen zwar immer noch im Mittelpunkt der Literatur, werden aber einer mitunter gnadenlosen Befragung unterzogen. Der Blick richtet sich nicht auf das behauptete Heile, sondern auf die Brüche darin. Themen, die im Naturalismus erstmals auftauchten, wie psychische und physische Störungen, Traumatisierung, Alkoholismus, verschiedene Formen der Sexualität, das Interesse für die gesellschaftlichen Außenseiter, Sprachlosigkeit usw., werden wieder aufgegriffen. Der Blick des Schreibenden entzieht sich dem Einfluss kirchlicher Autorität und gesellschaftlicher Verbindlichkeiten und wird dadurch gewissermaßen zu einem politischen Korrektiv.

67. Ebd., S. 68.

68. Ebd., S. 86. 